\title{
Data-driven Approach for Modeling the Thermal Dynamics of Residential Buildings Using a PieceWise ARX Model
}

\author{
Mohammed-Hichem Benzaama, Lala Rajaoarisoa, Balsam Ajib, Stéphane Lecoeuche \\ IMT Lille Douai, Univ. Lille, Unité de Recherche en Informatique Automatique, \\ F-59000, Lille, France
}

\begin{abstract}
This work presents a data-driven approach to identify a PieceWise affine Auto-Regressive eXogenous (PWARX) model, in order to evaluate a residential building's thermal behavior. The PWARX model is a PieceWise affine dynamic system that can model a large number of physical processes such a residential building. Indeed, the building is considered as a complex physical process influenced by several scenarios related to external solicitations and occupants by non linear complex system. For instance, presence or absence of solar radiation, presence or absence of occupants, and switch ON or switch OFF of the heating supply will lead to a variety of thermal behaviors. The PWARX model can identify these nonlinear dynamics using a set of ARX submodels with the same model structure, and each ARX sub-model represents a scenario or configuration. So this paper presents a data-driven approach to identify the PWARX model based on experimental data. Experimental data are collected for different rooms with different heating sequences, orientations and levels of a residential building located in Douai (France). The approach of the experimental validation consists to (i) determine the number of submodels in order to have the best fitting between the measured and estimated output, (ii) associate the data of each affine sub-model respectively, (iii) and finally, estimate each parameter vector associated to these submodels. The main objective of the study is then to find a structure for a set of switching thermal model to validate several configurations makes it possible to follow the experimental protocol and gets closer to reality.
\end{abstract}

\section{Introduction}

Building energy models have a paramount importance because they allow predicting and, then controlling, the energy demand. In particular, building energy modelling is used for (i) energy efficiency and optimal control; (ii) demand side management (DSM) strategies; (iii) fault detection and energy conservation (Ferracuti and Fonti (2017)). The dynamic model used to perform the predictions is of great importance and three categories regroup the numerous modeling approaches that have been considered (J.Siroky and Oldewurtel (2011)):

- white box models are based on physical knowledge of the system and thermal balance equations: these are often obtained through energy simulation software like EnergyPlus (Crawleya (2001)), TRNSYS (Benzaama and Lachi (2016)), etc;

- black box models use only measured input/output data and statistical estimation methods (e.g. (Cigler (2010) and Ferkl and Siroky (2010));

- grey box models, a mix of the first two categories: they use input/output data as well as some a priori knowledge on the system. A popular grey-box model is the equivalent RC networks (Wang and $\mathrm{Xu}$ (2006) and Afram and Fung (2018)).

Comparative study between these different models was done by Afram and Fung (2018). The results of this study allow to state on the fact the use of white box model often requires a computation time and a large number of inputs (Crawley and Hand (2008)), such as the composition of the structure of the building envelope. In some studies, this type of input might be difficult or even impossible to get (Kramer and van Schijndel (2013)). The other model categories allow determining the most efficient model for building integrated HVAC systems during the summer and wintering seasons for instance. Also, they show that the state-space models give a better accuracy compared to the other models, followed by ANN models and transfer functions and at the end process models and ARX (AutoRegressive eXogeneous) models.

Furthermore, several studies have been done on ARX models. As example reader can refer to Chen and Basu (2016) and Mechaqrane and Zouak (2004). In these works, the authors compared the ARX model with other models such as fractionnal-ARX (FARX), nonlinear ARX (NARX) and artificial neural network (ANN) models. Chen and Basu (2016) tested the FARX applied to energy systems integrated into buildings. The comparison between the FARX and ARX models shows that the FARX gives a good precision compared to the ARX models. The authors of Mechaqrane and Zouak (2004) and Soleimani-Mohseni and Thomas (2006) confirmed in their studies the under-performance of the ARX models compared to the models NARX and ANN models. The results of Mechaqrane and Zouak (2004) show that the NARX model performance was significantly better than to the ARX model for predicting of the indoor temperature of 
a residential building. Moreover, Soleimani-Mohsen Thomas (2006) concluded that the nonlinear ANN n outperformed the linear ARX model to estimate th erative temperature in buildings. To overcome the of the ARX model, several researchers developed a approach of ARX based on time interval and inpu dexed as Yun and Luck (2012). In this study, the au proposed a prediction model based on an autoregre scheme of orders 4 and indexed on 3 periods (day, t1 tion time and night) and five levels of ambient temper (4-3-5 ARX).

The inputs used in the models of Mechaqrane and $\mathrm{Z}$ (2004) and Yun and Luck (2012) are different with re to the models of Chen and Basu (2016) and Soleir Mohseni and Thomas (2006), which give a different s ture of model and it is often difficult to apply that in eral case (Touretzky and Patil (2015)). Moreover, th thors imposed the estimation of the input numbers wi doing a detailed analysis of their influence to validate the models. The local modes have also been arbitrarily chosen, without guarantees on the more accurate time-periods.

Despite the importance of data-driven approaches, datadriven prediction models have limitations (Kadir and Nora (2018)). Indeed, they may not perform well outside of their training range. Assumptions made by the learning algorithm have implications on the model's ability to cope with new data outside of the training data and whether it would generalize well beyond the training range or not. For example, a model that was trained by learning from a limited dataset (e.g., data collected from a small set of buildings) may not perform well outside of the training data (e.g., different types of buildings in terms of physical properties, operation strategies, weather conditions, occupant behavior, etc.).

\section{Paper contribution}

In this paper, we present a data-driven approach to identify a PWARX model, in order to evaluate a residential building's thermal behavior subject to multiple holdings. We also conduct a critical analysis of the inputs influence on the accuracy of the model to assess the quality of identified PWARX model. This is based on the influence order of each input on the structure of the model by taking into account several scenarios or configurations.

From the literature, only one work of (Ajib and Lefteriu (2018)) is available on experimental validation by the PWARX model to predict the future performance of the building under different operating conditions. The prediction of the temperature presented in this paper, allows to evaluate the energy consumption and energy control. Therefore, this study was conducted without taking into account the effect of the sensitivity analysis of the model. And yet, sensitivity analyses are an essential step to understand the overall inputs influence on the model parameter (i.e heating power $P w$, outside temperature $T_{0}$, outside humidity $H_{0}$ and solar radiation $R a$ ), on which the experimental plan effort should focus. This step allows configuring well the model, wins more computing time, im-

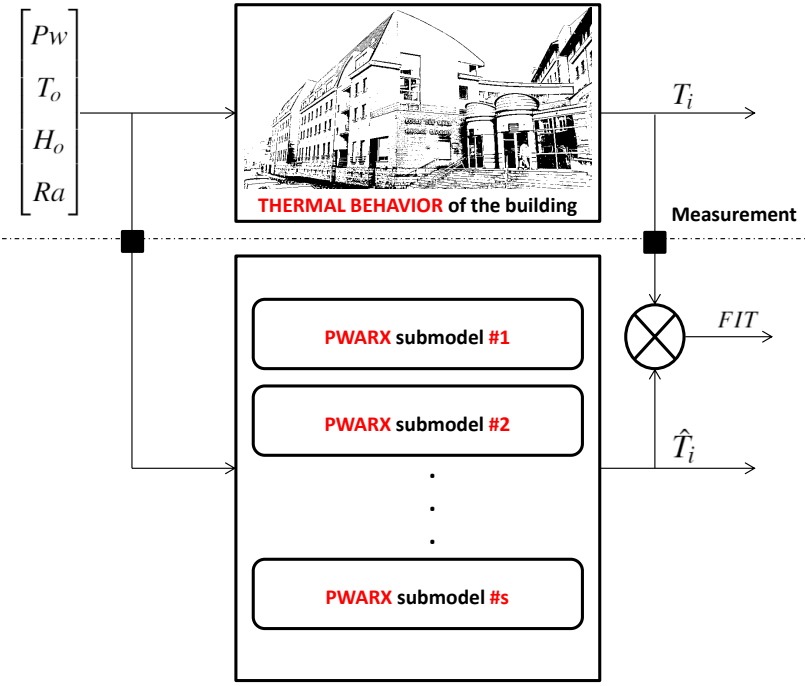

Figure 1: Flowchart for model identification.

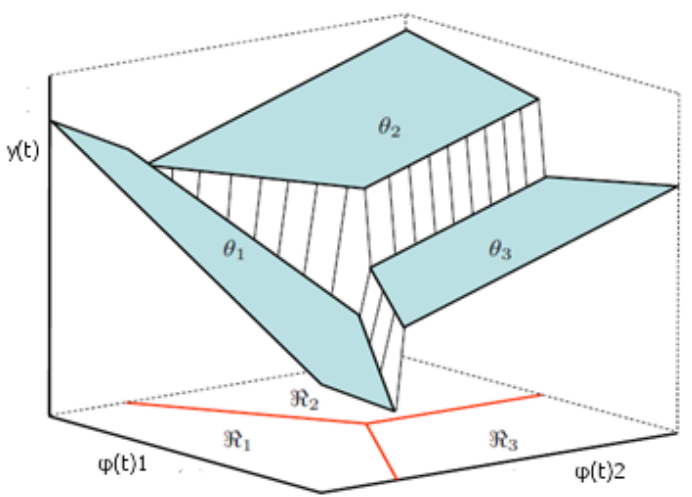

Figure 2: State-input domain partitioning.

prove accuracy and improve the knowledge on a PWARX model. Furthermore, the choice of the model order must be carefully defined in sensitivity analysis, because this can have a strong influence on the results. The evaluation of the most influential factors were then classified based on a criterion value $F I T$. This to rule on their influence, on the accuracy of the model and on the quality of identified parameters.

\section{PWARX model}

The PWARX model is a piecewise affine dynamic system, using a set of ARX submodels with the same model structure, that can model a large number of linear or nonlinear physical processes, including the residential buildings (figure 1).

The model is obtained by splitting the state-input domain represented by their parameters $\left\{\theta_{i}\right\}_{i=1}^{3}$ into a finite number of polyhedral regions (or configurations) $\left\{\mathfrak{R}_{i}\right\}_{i=1}^{3}$ (Figure 2) (Boukharouba (2011)).

PieceWise affine ARX (PWARX) models represent the input-output version of PWA models. They are defined by introducing the regression vector (for a Multiple Input 
Multiple Output:

$$
\varphi(t)=\left[y(t-1)^{T} \ldots y\left(t-n_{a}\right)^{T} \ldots u\left(t-n_{b}\right)^{T} \ldots u\left(t-n_{b}-n_{k}+1\right)^{T}\right]^{T} \quad(1
$$

Where, $n_{a}, n_{b}$ are the model orders and $n_{k}$ is the pure dela: between them. The input/output model of the PWARI model is represented by the following relation:

$$
y(t)=f(\varphi(t))+e(t)
$$

and $f$ is a piecewise affine map of the following form:

$$
f(\varphi(t))= \begin{cases}\theta_{1}^{T} \bar{\varphi}(t) & \text { if } \sigma(t)=1 \\ & \vdots \\ \theta_{s}^{T} \bar{\varphi}(t) & \text { if } \sigma(t)=s,\end{cases}
$$

where $\bar{\varphi}=\left[\begin{array}{ll}\varphi^{T} & 1\end{array}\right]^{T}$ is the extended regression vector $\sigma(t)$ is the switching rule defined by:

$$
\sigma(t)=i \text { if } \varphi(t) \in \mathfrak{R}_{i},
$$

and $\left\{\theta_{i}\right\}_{i=1}^{s}$ are the parameter vectors that define the sub models. $\left\{\mathfrak{R}_{i}\right\}_{i=1}^{s}$ represent a complete partition of the region $\mathfrak{R} \subset \mathbb{R}^{n}$, with $n=n_{e} n_{a}+\left(n_{b}+1\right)$, and each region is a convex polyhedron with

$$
\mathfrak{R}_{i}=\left\{\varphi \in \mathbb{R}^{n}: H_{i} \bar{\varphi} \preceq \mathbf{0}\right\}
$$

where $H_{i}$ and $\mathbf{0}$ are respectively a matrix of appropriate dimensions defining the limit of the region partitioning the set of regression vector and the null vector.

So, based on the input/output pair generated by the system described by (2), the identification of the PWARX model consist to: (i) determine the number of submodels in order to have the best fitting between the measured and estimated output, (ii) associate the data of each affine sub-model respectively, (iii)estimate each parameter vector $\theta_{i}$ associated to these submodels. Details about this identification procedure is given in the following section.

\section{Methodology}

To perform the identification of the PWARX model, we adopt the usual identification procedure defined by the following steps. Firstly, we design the experiment and the system necessary to collect the data. After that, we proceed to the determination of the model structure. And finally, we put in place an identification method and validation. It is globally illustrate in Figure 3.

\section{Experiment design and data collection}

Smart metering Having as main target the determination of a thermal model for a building without any particular prior knowledge on the system, we have developed in our laboratory a smart metering system that allows us to create a database rich in information about the thermal behavior of different buildings.

The architecture of the data collection system is illustrated in Figure 4. The supervisory and control acquisition data (SCADA) is based on different technologies. We use especially wireless sensors gathering by CLEODE ${ }^{T M}$ company to capture a number of information. Especially, to

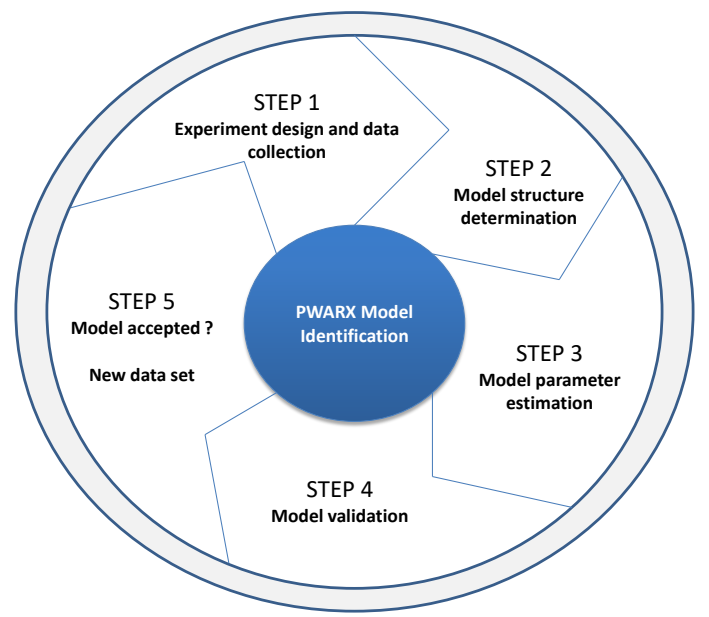

Figure 3: The necessary steps of system identification procedure.

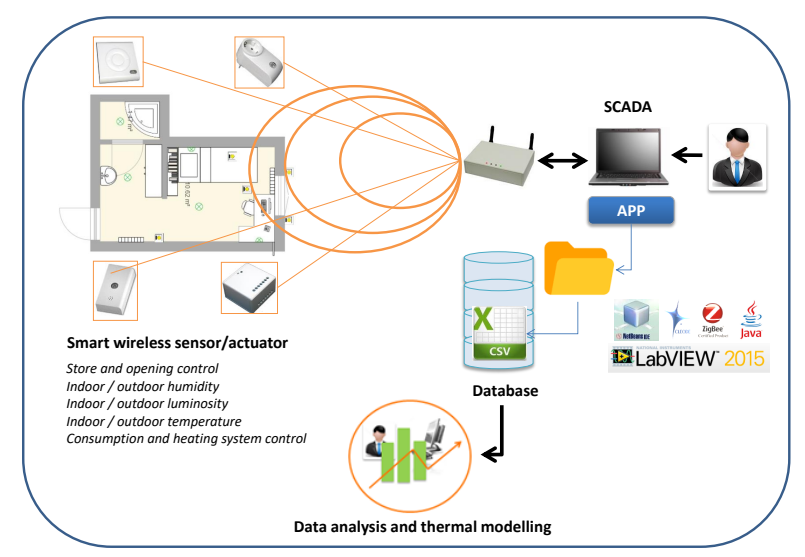

Figure 4: Global architecture of data collection system.

measure the indoor and outdoor temperature, indoor and outdoor humidity, solar radiation and incident solar radiation on the floor for different periods. A control system is used in this study to control the heating operation (ON/OFF) for several scenarios. Notice that, each sensor or actuator is scanned or controlled for a sampling time $T_{s}=2.4 \mathrm{~min}$.

Therefore, each measure is saved in database into CSV format thanks to applications that we have developed by our self using LABVIEW ${ }^{T M}$ and NetBeans ${ }^{T M}$ software's. The data collected is used after to compute the thermal model of the building studied, according to the planning detailed in the subsection below.

Experiment planning The planning of the experiments will be elaborated in such a way that one can appreciate the efficiency of a PWARX model for the prediction of the thermal behavior of a building.

Thus, experimental measurements have been made for different rooms of a residential building located in Douai (France) located at East/West for a different heating se- 
quence and various periods of acquisition. The purpose of the experimental plan is to disturb the thermal behavior of the indoor air by various factors such as the heating power and the climatic conditions (Solar radiation and outside temperature). The identified model will be tested on several case studies to see its ability to capture the thermal behavior of each case.

Indeed, the PWARX model can have different structures depending on the configuration considered.

\section{Parameter estimation of PWARX model}

The procedure used to estimate the different parameters of each sub-model can be describe into four steps. The first one is the initialization step. Knowing that the number of submodels is not known a priori, this step allows us to proceed to the creation of $q$ singletons classes with the $q$ regression available data. The second one is the reaffectation of data followed by the estimation of parameters of each sub-model. This step of the procedure consists in grouping the regression data (initially divided into $q$ classes) into a minimum number of disjoint classes. The third one is a verification step necessary for the stabilization of the data partition. And the last one is the validation step of the model thanks to a sensitivity analysis. Each step is detailed in the following paragraph.

Initialization In this part, we consider several $q$ submodels that correspond to $N$ classes, such that $C=$ $\left\{C_{1}, \cdots, C_{q}\right\}$ where each of them corresponds to one submodel defined by the parameter vector $\theta_{i}$ (Figure 4 ). The initial parameter vectors $\hat{\theta}_{1}^{0}, \cdots, \hat{\theta}_{q}^{0}$ is assigned, where $\hat{\theta}_{i}^{0}$ is computed by taking into account the data $x(i)$ of the class $C_{i}$ and its c nearest neighbor (c-cnn) and using the least square technique. Thus, the parameter vector $\hat{\theta}_{i}$ is calculated by least square technique while minimizing:

$$
V\left(\theta_{i}\right)=\frac{1}{N_{i}} \sum_{t=1}^{N_{i}}\left(y(t)-\theta_{i}^{T} \bar{\varphi}(t)\right)^{2}
$$

with $N_{i}$ is the number of points.

Data re-affectation and model estimation The main goal of this step is to minimize the number of classes that are initially divided into $q$ classes. For it, we minimize the Euclidean distance between each pair of data $(x(i), x(j))$ by:

$$
d_{i}^{j}=\|x(i)-x(j)\|
$$

Figure 5 illustrated the error between the measured output and the output of each sub-model.

Moreover, the ranking of the data is done according to the regression vector and the measured output as the following expression:

$$
x(i)=\left[\varphi(i)^{T}, \quad y(i)\right]^{T} .
$$

Thus, the data $x(i)$ will migrate towards the most representative classes according to a specific decision rule relative to the nearest neighbors (c-cnn) [16]:

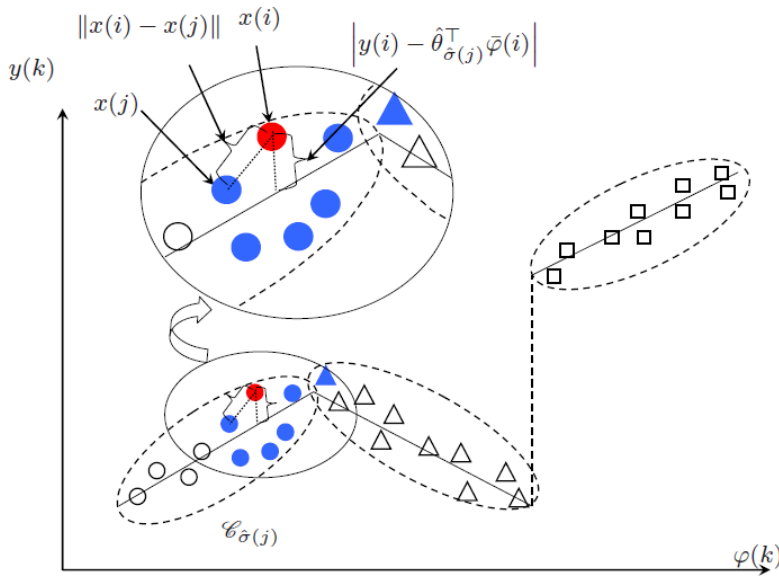

Figure 5: Data re-affectation effect.

$$
\begin{array}{r}
\varphi_{j}^{i}=\exp \left(-\alpha_{\hat{\sigma}(j)}\|x(i)-x(j)\|^{2}-\beta_{\hat{\sigma}(j)}(y(i)-\right. \\
\left.\left.\hat{\theta}_{\hat{\sigma}(j)}^{T} \bar{\varphi}(i)\right)\right)^{2}
\end{array}
$$

with $\hat{\theta}_{\hat{\sigma}(j)}^{T}$ is the parameter vector corresponding to the class $C_{\hat{\sigma}(j)}$. $\quad \alpha_{\hat{\sigma}(j)}$ and $\beta_{\hat{\sigma}(i)}$ are positive parameters. Thereafter, we need to update the class number $C=$ $\left\{C_{1}, \cdots, C_{\bar{s}}\right\}$ where $\bar{s} \leq q$. For the membership decision, we use the probability for which data point $\mathrm{x}(\mathrm{i})$ belongs to the class $C_{p}$ :

$$
P\left(x(i) \in C_{p}\right)=\frac{\sum_{j \mid x(j) \in\left\{\Gamma_{c}\left(x(i) \cap C_{q}\right)\right\}} \varphi_{j}^{i}}{\sum_{j=1}^{c} \varphi_{j}^{i}}, p \in\{1, \cdots, \bar{s}\}
$$

where $\bar{s}$ determine the persistent number of class in order to have the best fitting between the measured and estimated output.

Convergence criterion The convergence is based on the comparison between the earlier parameter vectors $\theta^{(r)}=\left[\hat{\theta}_{1}^{(r)}, \cdots, \hat{\theta}_{\bar{s}}^{(r)}\right]$ and the posterior parameter vectors $\theta^{(r+1)}$ where $r$ is the index of the iteration. Thus, the convergence criterion is writing as follows:

$$
\left\|\theta^{(r+1)}-\theta^{(r)}\right\| \leq v
$$

where $v$ is an arbitrary threshold defined by the user. Generally, one use $v=10^{-5}$.

Sensitivity analysis A recursive approach is used to test the influence of each input onto the model structures. In this paper, the input influence orders is obtained by comparing the FIT criterion value for different configurations. The FIT is in fact an indicator used here to measure the similarity between the measured output $y$ and the output reconstructed from the model $\hat{y}$. The FIT is given by the 
following expression:

$$
F I T=\left(1-\frac{\|\hat{y}-y\|}{\|y-\bar{y}\|}\right) \times 100 \%
$$

where $\bar{y}$ and $\hat{y}$ are respectively the sum and the estimate of the measures $y(t)$.

\section{Results and discussion}

This section presents the results of the sensitivity analysis of the model based on data collection. For this purpose, the case study and the various experimental parameters are presented. The switching between the heating modes is one of the parameters influencing on the validation. A comparison between the ARX and PWARX models according to different real heating mode is made to show the interest of a switched system identification technique such as PWARX to detect the different behaviors seen in a normal usage of a building zone.

\section{Case study and experimental data}

In this article, we present a case study on a room of $11 \mathrm{~m}^{2}$ of the university residence Lavoisier of the School of Mines, Douai in the north of France (Figure 6). This room is facing west on the 3 rd floor.

The choice of the west orientation and floor level was made to appreciate the effect of solar radiation on the thermal behavior of indoor air. The room is equipped with a heating system that works in a random.

Temperature sensor is installed to study the thermal behavior of the room. The experimental study was made from 14-02 to 17-02. the solar radiation received in this period is $100 \mathrm{~W} / \mathrm{m}^{2}$ as shown in Figure 7 .

The operation of the heating system is controlled in a random manner, which causes thermal excitation of the indoor air in a discrete way (Figure 8). the maximum heating power emitted by the electric convector is $1400 \mathrm{~W}$.

Figure 9 shows that the indoor temperature varies between 19 and $29^{\circ} \mathrm{C}$ for an outside temperature ranging between 2 and $10^{\circ} \mathrm{C}$.

\section{Sensitivity analysis}

Based on the training data from 07-02-2018 to 12-02-2018 East side, we present in this section the method for analyzing the effect of each parameter on the FIT. This method makes it possible to find the right model structure algorithm by making an analysis on the hyper parameters such as $n_{a}, n_{b}$, neighbour number $(c)$ and the inputs. In this article, the prediction of the indoor air temperature is sought. This output is influenced by several physical parameters (inputs) such as, outside temperature, solar radiation and heating power.

The results in figure 10 show that the hyper parameters $n_{a}, n_{b}$, and $C$ have a significant influence on the FIT. For the inputs "Outside temperature, solar radiation, heating power", the best estimate of the model corresponds to $n_{a}=n_{b}=2$ and $c=200$ classes (FIT $=78.48 \%$ ).

Figure 11 show the effect of each input on the Fit for the

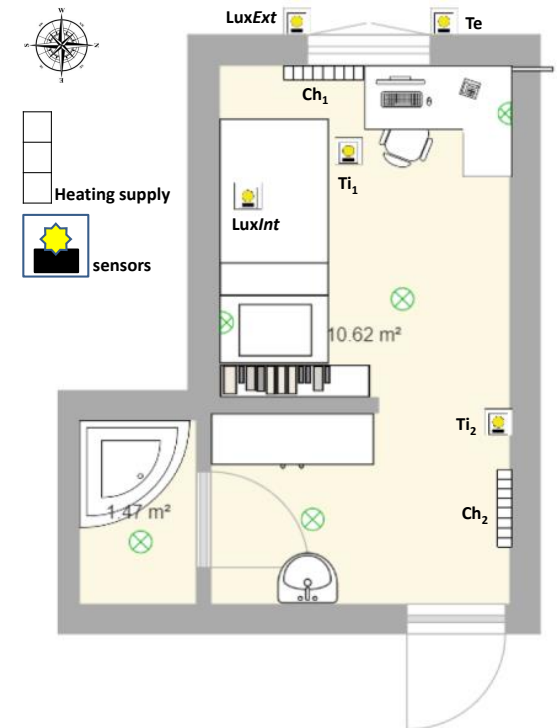

Figure 6: Student room configuration within sensor location.

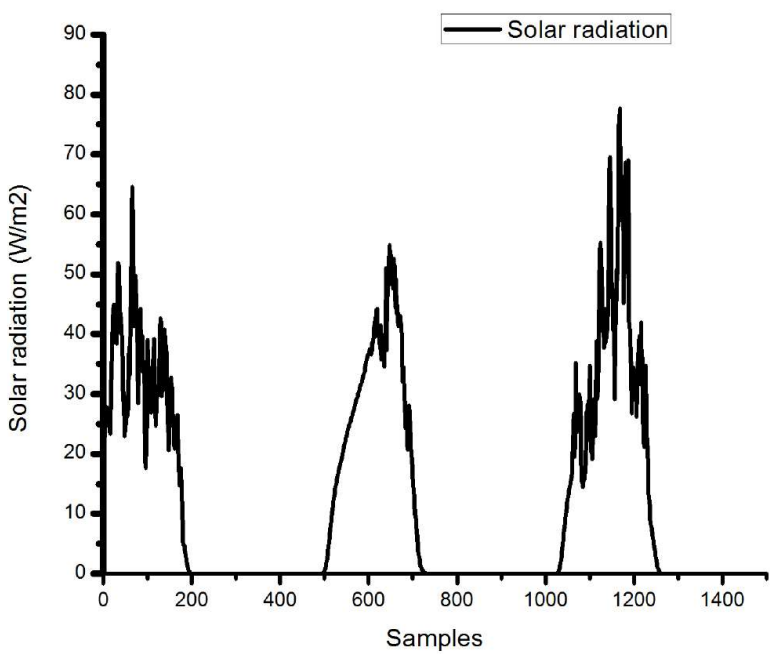

Figure 7: Solar radiation.

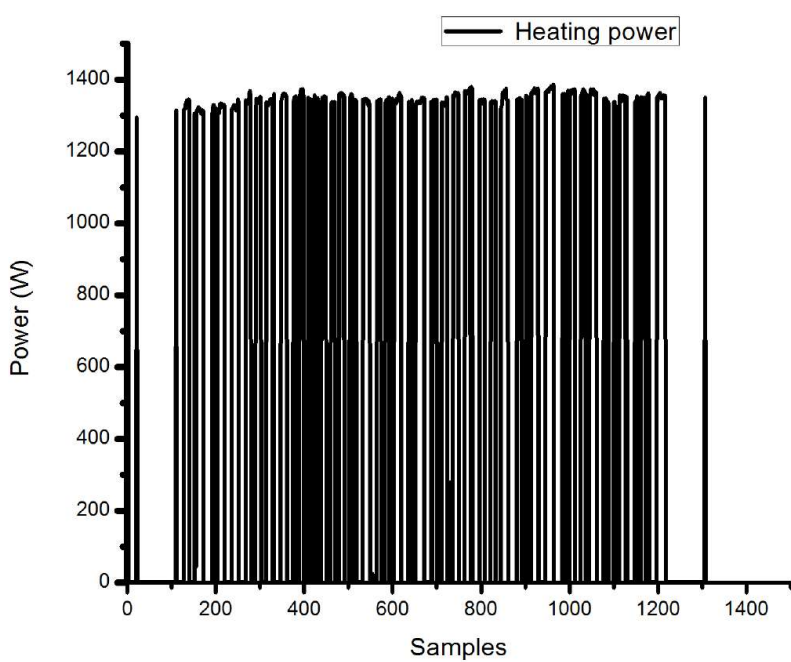

Figure 8: Heating scenario. 


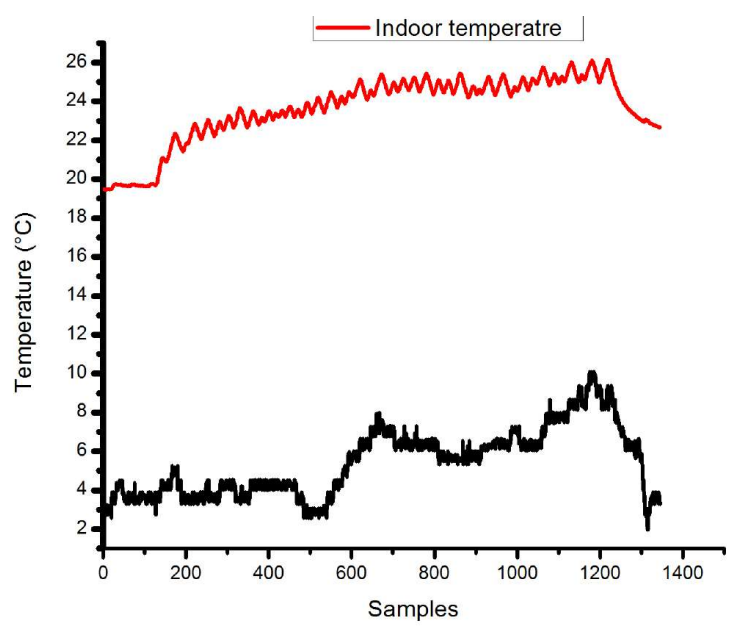

Figure 9: Indoor and outdoor air temperature measured.

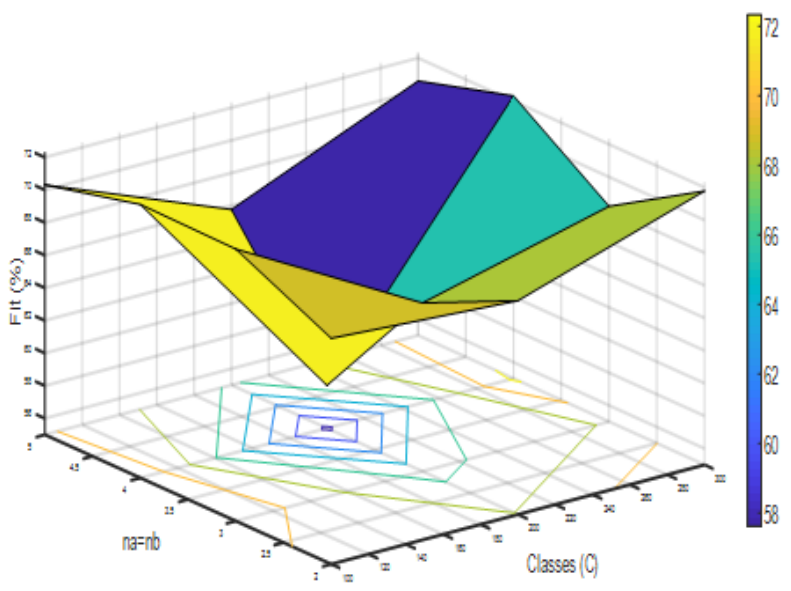

Figure 10: Effect of number of classes $(C)$ and number of discrete output and input $\left(n_{a}, n_{b}\right)$ on the FIT.

same hyper parameters $n_{a}=n_{b}=2$ and $\mathrm{C}=200$. The results show that absence of the outdoor temperature as input reduced the FIT by $8.1 \%$. On the other hand,the results show that the absence of the heating power in the model reduces the FIT by $53.87 \%$, while the absence of the solar radiation and heating power reduces the FIT by $63.21 \%$.

The analysis of the results shows that the best estimated model corresponds to structure: $n_{a}=n_{b}=2$ and $\mathrm{C}=200$ for the inputs "Outside temperature, Outside humidity, solar radiation, heating power" (Figure 12). The model found using the training data enable to validate other thermal behavior and measurement campaigns.

Based on the sensitivity analysis, we could give a recommendations on which the data collection effort should focus. The FIT may be changed according to the uncertain parameters. This change can affect the indoor air temperature predicted. Sensitivity analysis should give a capacity to structure well the model in terms of data and inputs which increases the accuracy and decreases the calculation time.

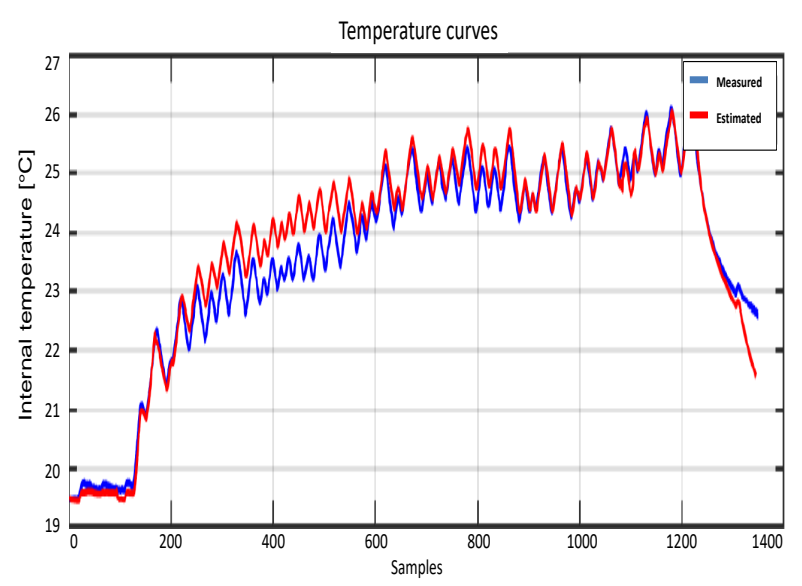

Figure 11: Training data (East Side $1^{\text {st }}$ floor).

Table 1: Switching conditions.

\begin{tabular}{|c|c|c|c|}
\hline Parameters & $P w$ & $R a$ & $T_{0}$ \\
\hline Mode 1 & ON & $>2.9 \mathrm{~W} / \mathrm{m}^{2}$ & $>4^{\circ} \mathrm{C}$ \\
\hline Mode 2 & OFF & $<2.9 \mathrm{~W} / \mathrm{m}^{2}$ & $>4^{\circ} \mathrm{C}$ \\
\hline Mode 3 & OFF & $<2.9 \mathrm{~W} / \mathrm{m}^{2}$ & $<4^{\circ} \mathrm{C}$ \\
\hline
\end{tabular}

The estimation of different thermal behavior and validation

The PWARX model has been compared with the experimental results as well as with the ARX model for the case of the a room located at East. Figure 13 and 14 show the validation of the indoor air temperature and the operation modes. The results show that the PWARX model is the best for prediction with a $F I T=78.48 \%$ compared to ARX model with a FIT $=41.67 \%$. This is due by the fact PWARX model estimates accurately the real temperature according to different possible scenarios influencing the thermal behavior of the room. The results show that the PWARX model is closer to reality compared to a standard ARX model.

Three different modes of thermal behavior have been observed (Figure 14). The first sub-model 1 corresponds to the configuration using the heating system $(\mathrm{ON})$, outdoor temperature greater than $4^{\circ} \mathrm{C}$ and presence of solar radiation (greater than $2.19 \mathrm{~W} / \mathrm{m}^{2}$ ). The second sub-model corresponds to the configuration heating OFF, outdoor temperature greater than $4^{\circ} \mathrm{C}$ and solar radiation less than $2.19 \mathrm{~W} / \mathrm{m}^{2}$, while the third sub-corresponds to the configuration heating OFF, outdoor temperature less than $4^{\circ} \mathrm{C}$ and solar radiation less than than $2.19 \mathrm{~W} / \mathrm{m}^{2}$ as shown in the table 1.

The PWARX model has been tested for another measurement campaign such as a room located in 3rd floor facing west (Figure 15). The thermal behavior of this room is different compared to the training period (orientation, heating scenarios and weather condition). Good agreement is shown in Figure 14 between the experimental results and the prediction with a $F I T=76.66 \%$, which shows that the structure of the estimated model is adapted both to East 


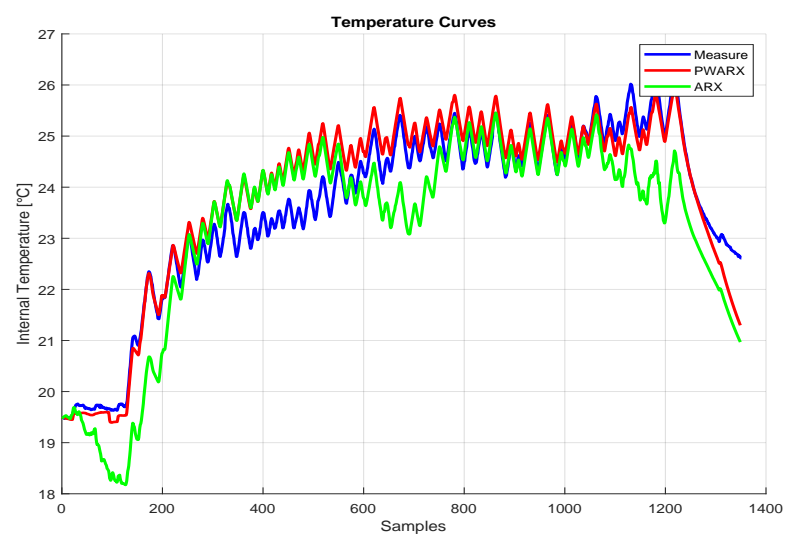

Figure 12: Indoor air temperature validation: Comparison between ARX (green solid line) and PWARX (red solid line) models.

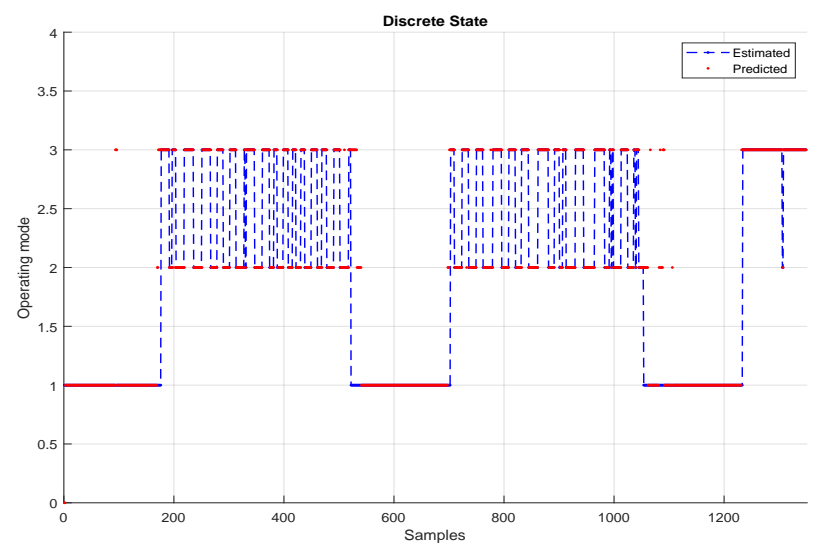

Figure 13: Operation mode validation: estimated (blue dashed line) and predicted (red dot line) operating mode.

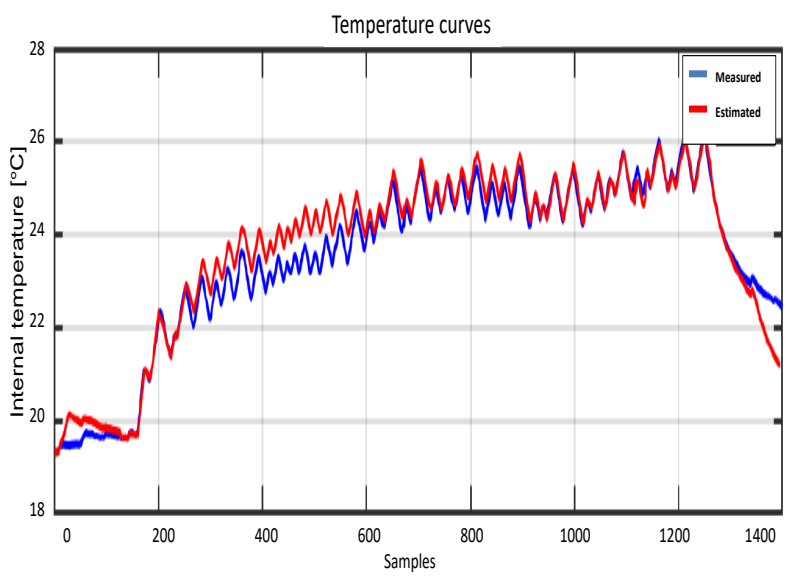

Figure 14: Validation of Temperature (West Side, 3rd Floor $), F I T=77.66 \%(14-02 / 17-02)$. and West orientation.

\section{Conclusion}

This article proposes a new data driven approach for building thermal modeling based on a piecewise ARX model. This approach takes into account the parameters influencing thermal behavior such as the location, the orientation of the building and the mode of operation of the heating source. The results show that the PWARX model allows to validate any configuration using a single model structure with the minimum of inputs compared to other models. The results of the structure analysis show that, the estimation of the model depends on several factors such as; the number of past input and output $\left(n_{a}, n_{b}\right)$, neighbour number and the nature of the physical magnitude imposed as input. We conclude that, solar radiation, heating power and outside humidity are indispensable for the identification of the model structure. The results of this paper show that the model is suitable for any orientation and floor levels with a random operation of the heating source, which facilitates the task of the building technician. Hence, future works will be directed to test this approach for predicting energy performance certificates for existing buildings, to minimize the energy consumption of the HVAC system.

\section{Acknowledgement}

This work was supported by the European project "Shine". A project granted by Interreg 2 Seas and the European Regional Development Fund.

\section{References}

Afram, A. and A. S. Fung (2018). Development and performance comparison of low-order black-box models for a residential hvac system. Journal of Building Engineering 15, 137-155.

Ajib, B. and S. Lefteriu (2018). Prediction of standardized energy consumption of existing buildings based on hybrid systems modeling and control. In 2018 IEEE Conference on Decision and Control (CDC).

Benzaama, M. and M. Lachi (2016). Study of the effect of sun patch on the transient thermal behaviour of a heating floor in algeria. Applied Energy 133, 257-270.

Boukharouba, K. (2011). Modelisation et classification de comportements dynamiques des systèmes hybrides. In Universite Lille.

Chen, L. and Basu (2016). Fractional order models for system identification of thermal dynamics of buildings. Energy and Buildings 133, 381-388.

Cigler, J. (2010). Csubspace identification and model predictive control for buildings. In 11th International Conference on Control, Automation, Robotic sand Vision.

Crawley, D. and B. Hand (2008). Contrasting the capabilities of building energy performance simulation programs. Building and Environnement 43, 661-673. 
Crawleya, B. (2001). Ecreating a new-generation building energy simulation program. In American Solar Energy SOC, The American Institute of Architects.

Ferkl, L. and J. Siroky (2010). Ceiling radiant cooling: comparison of armax and subspace identification modelling methods. Building and Environment 45, 205212.

Ferracuti, F. and A. Fonti (2017). Data-driven models for short-term thermal behaviour prediction in real buildings. Applied Energy 04, 1375-1387.

J.Siroky and F. Oldewurtel (2011). Experimental analysis of model pre-dictive control for an energy efficient building heating system. Applied Energy 88, 30793087.

Kadir, A. and M. E.-G. Nora (2018). A review of datadriven building energy consumption prediction studies. Renewable and Sustainable Energy Reviews 81, 11921205.

Kramer, R. and van Schijndel (2013). Inverse modeling of simplified hygrothermal building models to predict and characterize indoor climates. Building and Environnement $68,87-99$.

Mechaqrane, A. and M. Zouak (2004). comparison of linear and neural network arx models applied to a prediction of the indoor temperature of a building. Neural Computing and Applications 13, 32-37.

Soleimani-Mohseni, M. and Thomas (2006). Estimation of operative temperature in buildings using artificial neural networks. Energy and Buildings 38, 635-640.

Touretzky, C. and R. Patil (2015). Building-level power demand forecasting framework using building specific inputs: Development and applications. Applied Energy 147, 466-477.

Wang, S. and X. Xu (2006). Simplified building model for transient thermal performance estimation using gabased parameter identification. International Journal of Thermal Sciences 45, 419-432.

Yun, K. and Luck (2012). Building hourly thermal load prediction using an indexed arx model. Energy and Buildings 54, 225-233. 